\title{
The electronic spectrum of fullerenes from the Dirac equation
}

\author{
J. González ${ }^{\mathrm{a}, 1}$, F. Guinea ${ }^{\mathrm{b}, 2}$ and M.A.H. Vozmediano ${ }^{\mathrm{c}, 3}$ \\ a Instituto de Fisica Fundamental, CSIC, Serrano 123, 28006 Madrid, Spain \\ b The Harrison M. Randall Laboratory of Physics, The University of Michigan, Ann Arbor, \\ MI 48109, USA \\ c The Institute for Advanced Study, Princeton, NJ 08540, USA
}

Received 25 August 1992

Accepted for publication 24 May 1993

\begin{abstract}
The electronic spectrum of sheets of graphite (plane honeycomb lattice) folded into regular polyhedra is studied. A continuum limit valid for sufficiently large molecules and based on the tight-binding approximation is derived. It is found that a Dirac equation describes the flat graphite lattice. Curving the lattice by insertion of odd-numbered rings can be mimicked by coupling effective gauge fields. In particular the $\mathrm{C}_{60}$ and related molecules are well described by the Dirac equation on the surface of a sphere coupled to a color monopole sitting at its center.
\end{abstract}

\section{Introduction}

Fullerene molecules [1] are carbon cages which appear in the vaporization of graphite. They have become a source of great fun for physicist in different areas due to their curious properties and the relative ease in which they can now be synthesized and manipulated. One of their most beautiful features from a formal point of view is their geometrical character and the exciting possibility of producing them in all sorts of geometrical shapes having as building blocks sections of the honeycomb graphite lattice. The more abundant of them, the $\mathrm{C}_{60}$ molecule nicknamed "bucky ball", is also the most spherical. The sixty carbon atoms are placed at the vertices of a truncated icosahedron, obtained after replacing each of the icosahedron vertices by a regular pentagon. The shape of the $\mathrm{C}_{60}$ molecule is then that of a soccer ball, consisting of 12 pentagons and 20 hexagons. This molecule has shown to possess striking magnetic and electric properties, the most important of which is the superconducting nature of its alkaline compounds. In the process of graphite vaporization, there appear together with $\mathrm{C}_{60}$ a full set of

1 E-mail: emgonzalez@iem.csic.es

${ }^{2}$ On leave from: Instituto de Ciencias de Materiales, CSIC, Cantoblanco, 28050 Madrid, Spain.

${ }^{3}$ Present address: Instituto de Física Fundamental, CSIC, 28006 Madrid, Spain. 


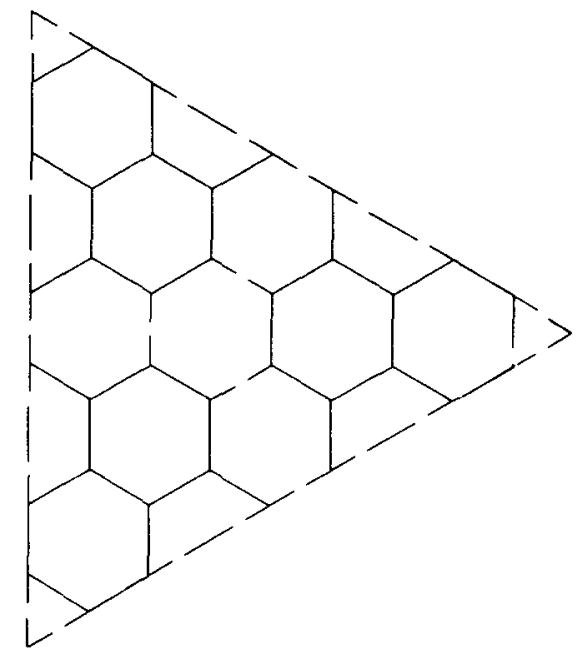

Fig. 1. Generic triangular block for honeycomb lattices folded on the tetrahedron, the octahedron and the icosahedron.

family members of various categories. Some are slightly deformed, as the $\mathrm{C}_{70}$ whose shape is more like an american football - an elliptical deformation-, some others are directly related with $\mathrm{C}_{60}$ in the sense that they are bigger but have the symmetry group of the icosahedron. These larger molecules may be imagined as built from triangular pieces of the honeycomb lattice of the type shown in fig. 1 . When the triangles are assembled as the faces of the icosahedron we end up with a lattice which has constant coordination number for all the sites. The first molecules of this series from the $\mathrm{C}_{60}$, namely the $\mathrm{C}_{240}$ and the $\mathrm{C}_{540}$ molecules, have already been synthesized ${ }^{*}$. The honeycomb lattice is very interesting in the study of two-dimensional statistical models as a rather nontrivial tessellation of the plane. The combination of the honeycomb lattice with the truncated icosahedron structure together with the existence of larger molecules grown from the mother $\mathrm{C}_{60}$ opens a totally new field of research, namely this of two-dimensional statistical models on curved lattices. Moreover, the compact geometry of the icosahedron is not the only possibility to fold the honeycomb lattice. The triangular pieces of the type shown in fig. 1 can be matched to form a lattice with constant coordination number inscribed in the tetrahedron or the octahedron. It turns out that, for all regular polyhedra whose faces are equilateral triangles, one may devise a recursive procedure to build respective series of lattices with growing number of points and preserving the symmetry group of the original polyhedra.

The main purpose of the present article is to study the electronic properties of

* A nice description of the fullerenes and their most curious features can be found in ref. [2]. 
the series of molecules which, starting from $\mathrm{C}_{60}$, have the symmetry group of the icosahedron. Of the four valence electrons which each carbon atom has, three of them build up the $\sigma$-orbitals along the lattice links and are responsible for the elastic properties. The remaining $\pi$-orbitals give rise to the conducting properties of the molecules, which can be considered as peculiar lattices at half-filling. In the description of the electronic excitations we will rely on the tight-binding method, which translates the problem into that of finding the spectrum of the hopping hamiltonian for the fermion operators $a_{i}, a_{j}^{+}$,

$$
H=\gamma \sum_{\langle i, j\rangle} a_{i}^{+} a_{j},
$$

the sum running over nearest neighbors $i, j$ in the curved lattice. In order to make contact with experiment, two different $\gamma$ hopping parameters should be taken for pentagon links and for links belonging to pairs of hexagons. This is consistent with the fact that in the $\mathrm{C}_{60}$ molecule, for instance, the interatomic distance along a pentagon link is slightly greater than the distance between neighboring atoms in different pentagons. It has been pointed out, however, that any ratio of the two hopping parameters between 0.9 and 1.0 gives quite reasonable results [3]. It is important to stress that, in spite of neglecting the coulombic interaction between the electrons, the tight-binding approximation predicts energy levels which are in good quantitative agreement with the existing experimental results $[3,4]$.

In this paper we undertake the problem of diagonalizing (1) in two steps. We first investigate the plane honeycomb lattice and we then fold it to form the given polyhedron. The pure graphite sheet is already very interesting in its own. The more important characteristic we find is that, instead of having a Fermi line, it has a finite set of isolated Fermi points when studied at half-filling. This is the physically interesting situation for carbon lattices as discussed in sect. 2 . This is the basis which allows us to build a local field theory to describe the low-energy excitations of the electronic hamiltonian about each of the Fermi points. The geometry and coordination of the lattice - it is in fact made of two interpenetrating triangular sublattices - determines the field theory to be that of a two-component Dirac spinor, while the existence of two independent Fermi points doubles the spectrum.

Next we come to the study of the folded lattices, having in mind in particular the lattices of the three basic regular polyhedra: tetrahedron, octahedron and icosahedron. All of them produce by simple truncation a lattice that can then be grown indefinitely the same way as the $\mathrm{C}_{60}$ does. Being regular polyhedra, all of them can be flattened off on the plane in a certain way so that we are back to the study of the honeycomb lattice in a portion of the plane with rather nontrivial boundary conditions. Notice that this approach, by emphasizing the translational invariance of the problem, is orthogonal to the ones that 
use group-theoretical methods based on the rotational symmetry of the molecule [4]. When this procedure is applied to the tetrahedron the first thing that we observe is that its conical singularities (vertices), although truncated, generate lines of frustration in the lattice. More concretely, its two triangular sublattices are interchanged by the boundary conditions. As a consequence of this, we learn about the interchange of Fermi points (or of the atoms in the basis of the Bravais lattice) that takes place in the tetrahedron as well as in the icosahedron *. The effect of frustration is then twofold, since on one side we have curvature and on the other side we have to couple the formerly independent Dirac spinors to produce the mentioned interchange. For that we treat the two Dirac spinors as the two components of an $\mathrm{SU}(2)$ color doublet. Each vertex is traversed by a quantum of color magnetic flux mimicking the interchange of color amplitudes induced by the vertices of the polyhedron. We are able in this way to make contact with the phenomenological study presented in ref. [5]. There the effect of the magnetic field was smoothed over the sphere by considering a monopole sitting at its center, and the simplified model for the icosahedral $\mathrm{C}_{60}$ with an abelian monopole of charge $\frac{3}{2}$ reproduced the observed low-energy spectrum. The refinement presented here represents a qualitatively better understanding of the problem and allows us to predict the correct value of the monopole charge.

The presentation of the article goes as follows: in sect. 2 we study in full detail the plane honeycomb lattice, perform the tight-binding approximation and extract the continuum limit. In sect. 3 we describe the technique for solving the free-field hamiltonian in the honeycomb lattice folded on regular polyhedra. The case of the grown tetrahedron with 48 lattice points is worked out as an explicit example. In sect. 4 we explain in detail the phenomenological model proposed to compute the spectrum of large icosahedral molecules. We will justify the validity of the continuum limit for the fullerene molecules, discuss the role played by the peculiar geometry of the honeycomb lattice, and write the appropriate Dirac equation. In sect. 5 we will write down a summary of the main points developed through the paper highlighting the more important issues and will discuss its implications and prospects.

\section{The planar honeycomb lattice}

We will see in what follows that the tight-binding approximation applied to the computation of the electronic spectrum in periodic potentials leads to the equivalent problem of the spectrum of a free-field theory on a lattice. We will focus on the resolution of the honeycomb lattice on the two-dimensional plane.

In solid-state applications one is interested in periodic potentials which cor-

* We note by passing that such interchange does not take place in the octahedron which will be treated in a different publication. 
respond to a given disposition of the atoms in a crystal. The set of discrete translations under which the potential $V(\boldsymbol{r})$ is invariant can be generated by independent transformations $\boldsymbol{T}_{1}$ and $\boldsymbol{T}_{2}$ (in two dimensions) so that

$$
V\left(\boldsymbol{r}+p \boldsymbol{T}_{1}+q \boldsymbol{T}_{2}\right)=V(\boldsymbol{r}) \quad \forall p, q \in \mathbb{Z} .
$$

The statement of Bloch theorem is that the energy eigenfunctions of the quantum problem can be expressed as

$$
\Psi_{n \boldsymbol{k}}(\boldsymbol{r})=\exp (i \boldsymbol{k} \cdot \boldsymbol{r}) u_{n \boldsymbol{k}}(\boldsymbol{r}),
$$

with $u_{n k}(\boldsymbol{r})$ being a periodic function

$$
u_{n k}\left(\boldsymbol{r}+p \boldsymbol{T}_{1}+q \boldsymbol{T}_{2}\right)=u_{n k}(\boldsymbol{r}) \quad \forall p, q \in \mathbb{Z} .
$$

In the above expression $n$ stands for the band label, while $\boldsymbol{k}$ labels different states in a band. A way of exploiting the content of Bloch theorem is to write the energy eigenfunctions (3) in the form of the so-called Wannier functions

$$
\Psi_{n \boldsymbol{k}}(\boldsymbol{r})=\frac{1}{\sqrt{N}} \sum_{i} \exp \left(i \boldsymbol{k} \cdot \boldsymbol{r}_{i}\right) \phi_{n}\left(\boldsymbol{r}-\boldsymbol{r}_{i}\right)
$$

where the sum runs over all the lattice points

$$
\boldsymbol{r}_{i}=p_{i} \boldsymbol{T}_{1}+q_{i} \boldsymbol{T}_{2}, \quad p_{i}, q_{i} \in \mathbb{Z} .
$$

It can be shown that (5) is a Bloch wavefunction and, vice versa, that an eigenfunction with the property (3) can always be cast in the form (5) (see for instance ref. [6]).

The simplest instance in applying the tight-binding method occurs when there is no significant mixing between states belonging to different bands. Then one can insert one linear combination (5) in the computation of the energy eigenvalues. Since the energy eigenfunctions are always expressible in the form (5), we may take advantage of the variational approach to conclude that the energy levels are given by

$$
E_{\boldsymbol{k}}=\frac{\int \mathrm{d}^{2} \boldsymbol{r} \bar{\Psi}_{\boldsymbol{k}} H \Psi_{\boldsymbol{k}}}{\int \mathrm{d}^{2} \boldsymbol{r} \bar{\Psi}_{\boldsymbol{k}} \Psi_{\boldsymbol{k}}}=\frac{\sum_{i} \exp \left(i \boldsymbol{k} \cdot \boldsymbol{r}_{i}\right) \int \mathrm{d}^{2} \boldsymbol{r} \bar{\phi}(\boldsymbol{r}) H \phi\left(\boldsymbol{r}-\boldsymbol{r}_{i}\right)}{\sum_{i} \exp \left(i \boldsymbol{k} \cdot \boldsymbol{r}_{i}\right) \int \mathrm{d}^{2} \boldsymbol{r} \bar{\phi}(\boldsymbol{r}) \phi\left(\boldsymbol{r}-\boldsymbol{r}_{i}\right)} .
$$

In physical situations in which the orbitals $\phi$ are localized around each lattice site, it may be appropriate to approximate the numerator by integrals involving only nearest-neighbor orbitals, which is the essence of the tight-binding method. In practice, it is also reasonable to take the norm of the Wannier wavefunctions as constant, which we set to one by a proper normalization. This gives the result for the energy levels in tight-binding approximation

$$
E_{\boldsymbol{k}}=\int \mathrm{d}^{2} r \bar{\phi}(\boldsymbol{r}) H \phi(\boldsymbol{r})+\sum_{\substack{\text { nearest } \\ \text { neighbors }}} \exp \left(i \boldsymbol{k} \cdot \boldsymbol{r}_{i}\right) \int \mathrm{d}^{2} r \bar{\phi}(\boldsymbol{r}) H \phi\left(\boldsymbol{r}-\boldsymbol{r}_{i}\right),
$$




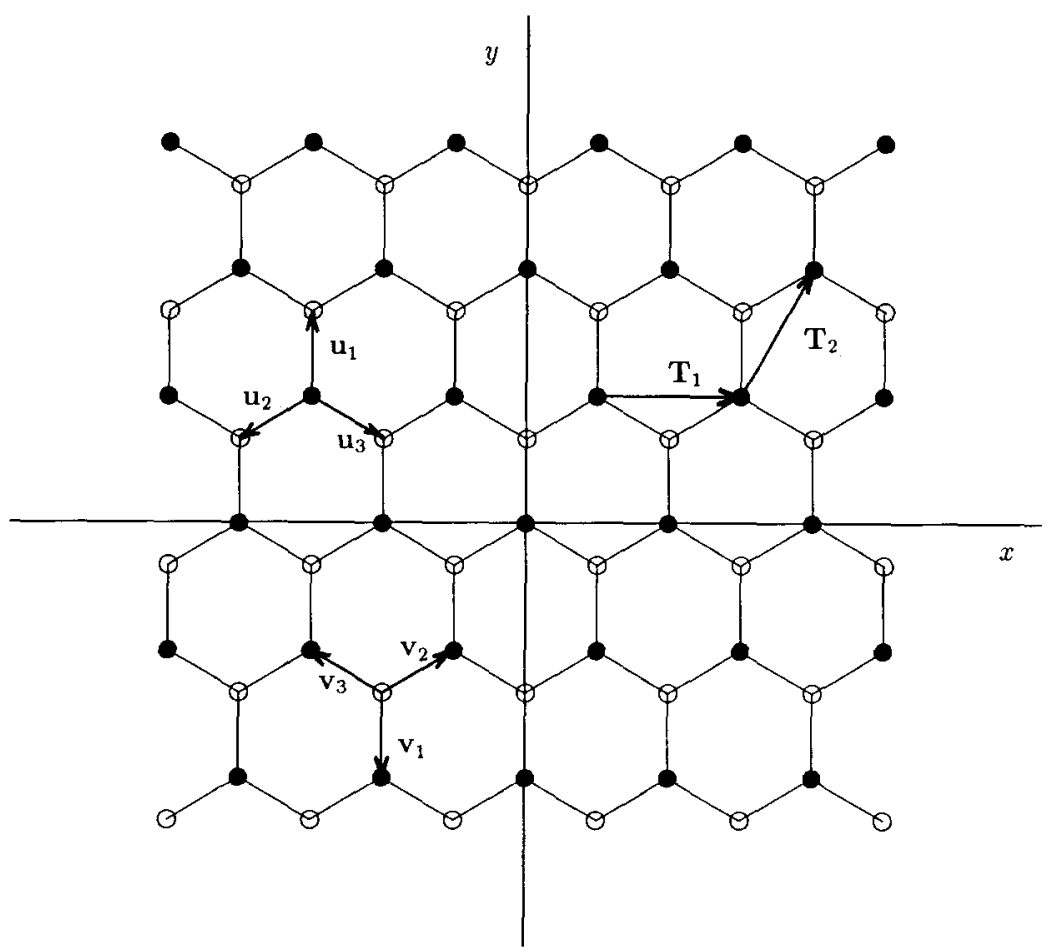

Fig. 2. The planar honeycomb lattice.

where the sum runs over all nearest neighbors $i$ of the origin.

The derivation we have made is appropriate to lattices which have only one atom per primitive cell, though the extension of the method to the general case is straightforward. For the lattice which is the object of our interest, the honeycomb lattice of graphite shown in fig. 2 , there are two atoms in the primitive cell. We may take as generators of the lattice the vectors

$$
\boldsymbol{T}_{1}=\sqrt{3} \boldsymbol{e}_{x}, \quad \boldsymbol{T}_{2}=\frac{1}{2} \sqrt{3} \boldsymbol{e}_{x}+\frac{3}{2} \boldsymbol{e}_{y}
$$

Then we need to place an atom at the origin of the primitive cell and another displaced at $\boldsymbol{d}=\boldsymbol{e}_{y}$, for instance, to produce the honeycomb lattice by repeated application of the generators. The wavefunctions $\phi$ may be thought of being composed of two identical orbitals $\phi_{\bullet}$ and $\phi_{\diamond}$ localized respectively around each of the two mentioned points of the primitive cell. We may exploit the variational freedom by considering an arbitrary linear combination of these two orbitals, which we write in the form

$$
\phi(\boldsymbol{r})=c_{\bullet} \phi_{\bullet}(\boldsymbol{r})+c_{0} \exp (i \boldsymbol{k} \cdot \boldsymbol{d}) \phi_{0}(\boldsymbol{r}-\boldsymbol{d}) .
$$

By introducing this expression into the energy functional we get, in tight-binding 
approximation,

$$
\begin{aligned}
E_{\boldsymbol{k}}= & \bar{c}_{\bullet} c_{\bullet} \int \mathrm{d}^{2} r \bar{\phi}_{\bullet}(\boldsymbol{r}) H \phi_{\bullet}(\boldsymbol{r})+\bar{c}_{\circ} c_{\circ} \int \mathrm{d}^{2} r \bar{\phi}_{\circ}(\boldsymbol{r}-\boldsymbol{d}) H \phi_{\circ}(\boldsymbol{r}-\boldsymbol{d}) \\
& +\bar{c}_{\bullet} c_{\circ} \sum_{j} \exp \left(i \boldsymbol{k} \cdot \boldsymbol{u}_{j}\right) \int \mathrm{d}^{2} r \bar{\phi}_{\bullet}(\boldsymbol{r}) H \phi_{\circ}\left(\boldsymbol{r}-\boldsymbol{u}_{j}\right) \\
& +\bar{c}_{\circ} c_{\bullet} \sum_{j} \exp \left(i \boldsymbol{k} \cdot \boldsymbol{v}_{j}\right) \int \mathrm{d}^{2} r \bar{\phi}_{\circ}(\boldsymbol{r}-\boldsymbol{d}) H \phi_{\bullet}\left(\boldsymbol{r}-\boldsymbol{d}-\boldsymbol{v}_{j}\right),
\end{aligned}
$$

where $\left\{\boldsymbol{u}_{j}\right\}$ is a triad of vectors of link length pointing respectively in the direction of the nearest neighbors of a $\bullet$ point, and $\left\{\boldsymbol{v}_{j}\right\}$ the triad made of their respective opposites (see fig. 2). We face the ideal situation in which neither the $\phi_{\bullet}$ - nor the $\phi_{\circ}$-orbitals are directionated over the two-dimensional plane. Then the symmetry of the problem imposes that

$$
\begin{array}{r}
\int \mathrm{d}^{2} r \bar{\phi}_{\bullet}(\boldsymbol{r}) H \phi_{\bullet}(\boldsymbol{r})=\int \mathrm{d}^{2} r \bar{\phi}_{\circ}(\boldsymbol{r}-\boldsymbol{d}) H \phi_{\circ}(\boldsymbol{r}-\boldsymbol{d})=\beta, \\
\int \mathrm{d}^{2} r \bar{\phi}_{\bullet}(\boldsymbol{r}) H \phi_{\circ}\left(\boldsymbol{r}-\boldsymbol{u}_{i}\right)=\int \mathrm{d}^{2} r \bar{\phi}_{\circ}(\boldsymbol{r}-\boldsymbol{d}) H \phi_{\bullet}\left(\boldsymbol{r}-\boldsymbol{d}-\boldsymbol{v}_{i}\right)=\gamma
\end{array}
$$

The variational problem for the honeycomb lattice becomes, then, the diagonalization of the quadratic form

$$
E_{\boldsymbol{k}}=\left(\bar{c}_{\bullet} \quad \bar{c}_{\circ}\right)\left(\begin{array}{cc}
\beta & \gamma \sum_{j} \exp \left(i \boldsymbol{k} \cdot \boldsymbol{u}_{j}\right) \\
\gamma \sum_{j} \exp \left(i \boldsymbol{k} \cdot \boldsymbol{v}_{j}\right) & \beta
\end{array}\right)\left(\begin{array}{l}
c_{\bullet} \\
c_{\circ}
\end{array}\right) .
$$

We will disregard in what follows the diagonal contribution in (14) as long as it is independent of $\boldsymbol{k}$. We will come back later to the band structure of the honeycomb lattice of graphite.

The approximations made by the tight-binding method reduce the problem, in practice, to that of a set of coupled oscillators on the lattice. This can be appreciated in the expression of the energy functional (14), in which what matters is essentially the coordination between the lattice sites. By application of the tight-binding method what we are doing actually is diagonalizing the hamiltonian

$$
H=\gamma \sum_{\langle i, j\rangle} a_{i}^{+} a_{j},
$$

where the sum is over pairs of nearest-neighbors atoms $i, j$ on the lattice and $a_{i}$ ,$a_{j}^{+}$are canonically anticommuting operators

$$
\left\{a_{i}, a_{j}\right\}=\left\{a_{i}^{+}, a_{j}^{+}\right\}=0, \quad\left\{a_{i}, a_{j}^{+}\right\}=\delta_{i j} .
$$

In fact, this problem can be solved by a variant of the method sketched above. 
We first form the eigenstate of $T_{1}$ and $T_{2}$

$$
\Psi=\sum_{i \bullet} c_{\bullet} \exp \left(i \boldsymbol{k} \cdot \boldsymbol{r}_{i}\right) a_{i}^{+}|O\rangle+\sum_{i \circ} c_{0} \exp \left(i \boldsymbol{k} \cdot \boldsymbol{r}_{i}\right) a_{i}^{+}|O\rangle,
$$

assigning different coefficients $c_{\bullet}$ and $c_{\circ}$ to black and blank points, respectively, as depicted in fig. 2. Under the action of the hamiltonian, however, black points are mapped into blank points and vice versa. We have, indeed,

$$
\begin{aligned}
H \Psi & =\gamma \sum_{i \bullet\langle i, j\rangle} \sum_{0} \exp \left(i \boldsymbol{k} \cdot \boldsymbol{r}_{j}\right) a_{i}^{+}|O\rangle+\gamma \sum_{i \circ} \sum_{\langle i, j\rangle} c_{\bullet} \exp \left(i \boldsymbol{k} \cdot \boldsymbol{r}_{j}\right) a_{i}^{+}|O\rangle \\
& =\gamma \sum_{j} \exp \left(i \boldsymbol{k} \cdot \boldsymbol{u}_{j}\right) \sum_{i \bullet} c_{0} \exp \left(i \boldsymbol{k} \cdot \boldsymbol{r}_{i}\right) a_{i}^{+}|O\rangle \\
& +\gamma \sum_{j} \exp \left(i \boldsymbol{k} \cdot \boldsymbol{v}_{j}\right) \sum_{i \circ} c_{\bullet} \exp \left(i \boldsymbol{k} \cdot \boldsymbol{r}_{i}\right) a_{i}^{+}|O\rangle .
\end{aligned}
$$

It is clear that the state (17) is an eigenvector of $H$ provided that the coefficients $c_{\bullet}$ and $c_{\circ}$ are solutions of the eigenvalue problem

$$
\left(\begin{array}{cc}
0 & \gamma \sum_{j} \exp \left(i \boldsymbol{k} \cdot \boldsymbol{u}_{j}\right) \\
\gamma \sum_{j} \exp \left(i \boldsymbol{k} \cdot \boldsymbol{v}_{j}\right) & 0
\end{array}\right)\left(\begin{array}{l}
c_{\bullet} \\
c_{\circ}
\end{array}\right)=E_{\boldsymbol{k}}\left(\begin{array}{l}
c_{\bullet} \\
c_{0}
\end{array}\right) .
$$

This is nothing but a different expression of the variational problem (14).

From (19) a straightforward computation gives the band of levels

$$
E_{k}= \pm \gamma \sqrt{1+4 \cos ^{2} \frac{1}{2} \sqrt{3} k_{x}+4 \cos \frac{1}{2} \sqrt{3} k_{x} \cos \frac{3}{2} k_{y}} .
$$

The structure of this band has very striking properties when considered at halffilling. This is the situation which has physical interest, since in the case of graphite each site of the honeycomb lattice yields one electron to the Fermi sea. Each level of the band may accommodate two states due to the spin degeneracy, and the Fermi level turns out to be at the midpoint of the band, $E_{k}=0$. Quite amazingly, the honeycomb lattice at half-filling has six isolated Fermi points, instead of a whole Fermi line. In the reciprocal lattice generated by

$$
\boldsymbol{K}_{1}=\frac{2 \pi}{\sqrt{3}} \boldsymbol{e}_{x}-\frac{2}{3} \pi e_{y}, \quad \boldsymbol{K}_{2}=\frac{4}{3} \pi \boldsymbol{e}_{y}
$$

the first Brillouin zone has as many momenta as primitive cells contains the original lattice. Such a primitive cell of the reciprocal lattice is an hexagon, as shown in fig. 3 . The only points which reach the Fermi level are the six vertices of the hexagon

$$
\begin{array}{ll}
k_{x}= \pm \frac{4 \pi}{3 \sqrt{3}}, & k_{y}=0, \\
k_{x}= \pm \frac{2 \pi}{3 \sqrt{3}}, & k_{y}= \pm \frac{2}{3} \pi .
\end{array}
$$




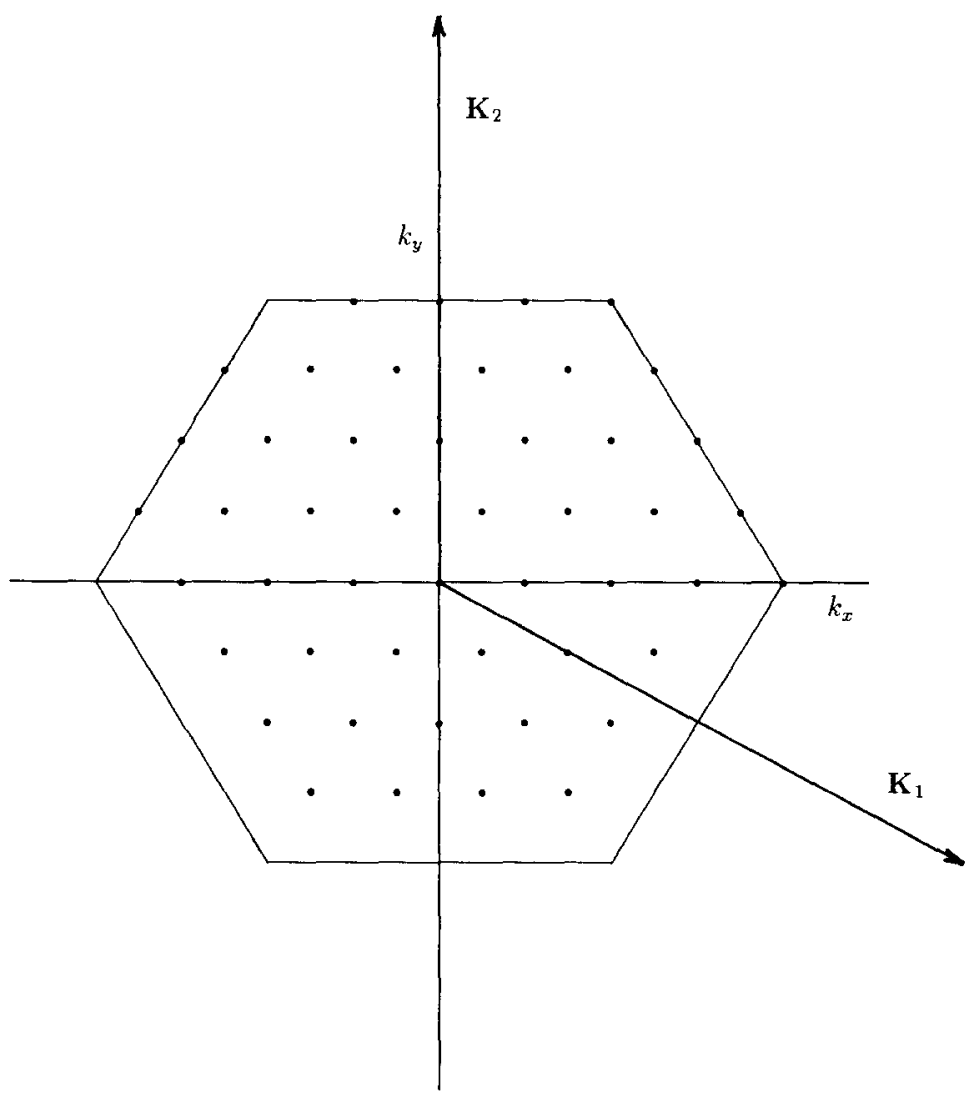

Fig. 3. The reciprocal lattice in momentum space. The hexagon depicted in the figure represents the first Brillouin zone. The dots stand for independent energy eigenstates of the lattice in fig. 5 .

It can be checked that these are the only roots of $E_{k}=0$. The representation of the lower branch of the band in fig. 4 illustrates the peculiar form of the Fermi sea. At last, the independent number of Fermi points is two, since any two momenta congruent by $\boldsymbol{K}_{1}, \boldsymbol{K}_{2}$ are just different labels of the same state.

The existence of a finite number of Fermi points at half-filling has important consequences in the description of the spectrum about the Fermi level. The low-energy excitations can be studied by taking the continuum limit at any two independent Fermi points. As long as the number of them is finite, the outcome is that a simple field theory suffices to describe the electronic spectrum of large honeycomb lattices. The continuum limit can be taken by naïve scaling of dimensionful quantities since we are dealing with a free theory. For this purpose we introduce a parameter $a$ measuring the link length and expand the $2 \times 2$ operator in (19) at any of two independent Fermi points. At the first Fermi point in (22), for instance, we have

$$
\boldsymbol{k}=\frac{4 \pi}{3 \sqrt{3}} e_{x}+\boldsymbol{\delta} \boldsymbol{k}
$$




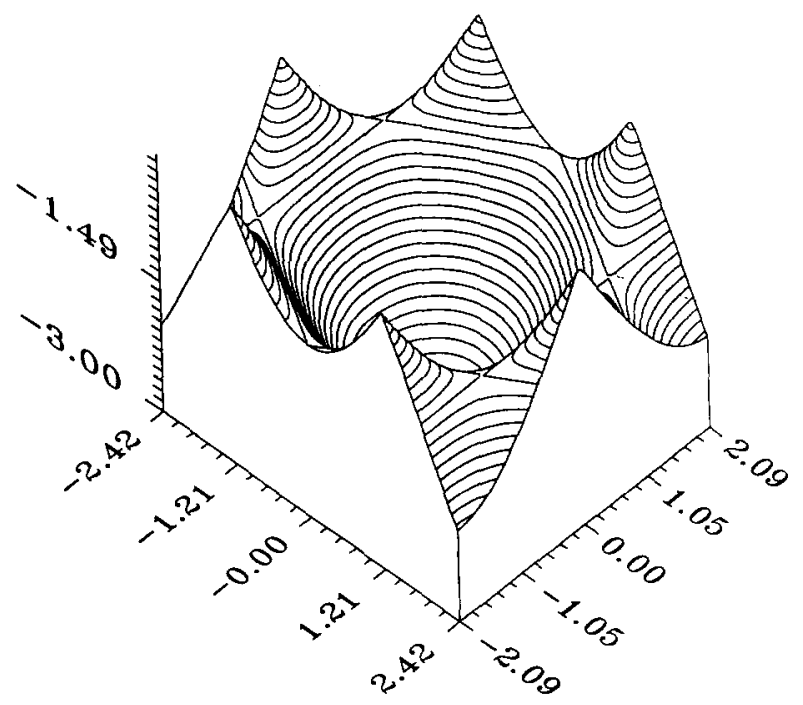

Fig. 4. Representation in $(E, \boldsymbol{k})$ space of the lower branch of the electronic dispersion relation $(\gamma=-1)$. The cusps appear at the six corners of the first Brillouin zone.

and

$$
\begin{aligned}
\mathcal{H} & \equiv\left(\begin{array}{cc}
0 & \gamma \sum_{j} \exp \left(i a \boldsymbol{k} \cdot \boldsymbol{u}_{j}\right) \\
\gamma \sum_{j} \exp \left(i a \boldsymbol{k} \cdot \boldsymbol{v}_{j}\right) & 0
\end{array}\right) \\
& \approx-\frac{3}{2} \gamma a\left(\begin{array}{cc}
0 & \delta k_{x}+i \delta k_{y} \\
\delta k_{x}-i \delta k_{y} & 0
\end{array}\right)+\mathrm{O}\left((a \delta k)^{2}\right) .
\end{aligned}
$$

The naïve scaling

$$
\lim _{a \rightarrow 0} \mathcal{H} / a=-\frac{3}{2} \gamma \boldsymbol{\sigma}^{\mathrm{T}} \cdot \boldsymbol{\delta} \boldsymbol{k}
$$

dictates the effective hamiltonian in the continuum limit, which turns out to be the Dirac operator in two dimensions. The same result is obtained at any of the six points in (22). Given the existence of two independent Fermi points, we conclude that the low-energy excitations of the honeycomb lattice at half-filling are described by an effective theory of two two-dimensional Dirac spinors. This result is at odds with the more standard continuum approximation to lattice theories in condensed matter physics, the effective mass theory. There, a quadratic dispersion relation at high-symmetry points of the Brillouin zone gives rise to an effective Schrödinger equation, with one parameter, the mass, chosen to reproduce the exact curvature. Only one-dimensional systems, and three-dimensional semiconductors with the diamond structure and no gap, are known to give rise to the Dirac equation. 


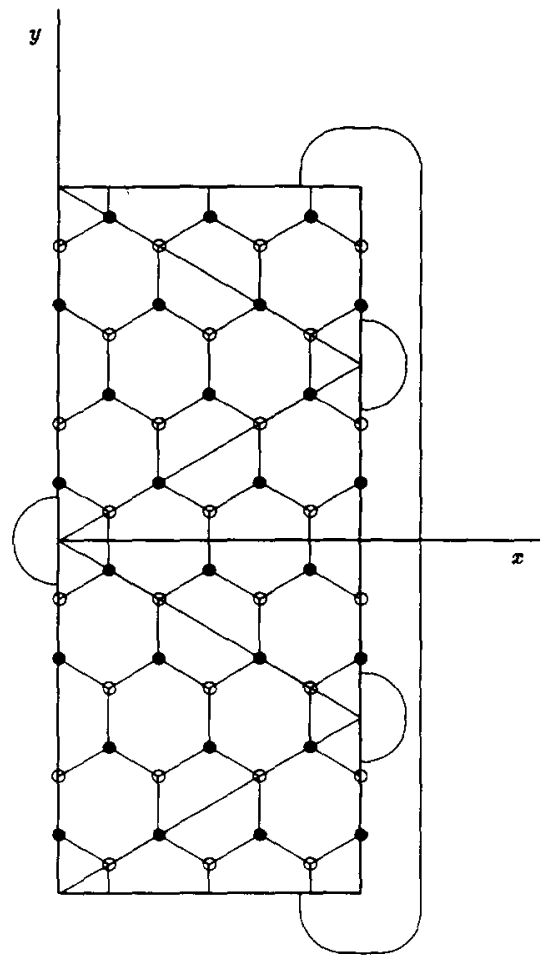

Fig. 5. Curved honeycomb lattice unfolded on the plane. The outer lines indicate the identifications between boundary segments which embed the lattice on the tetrahedron.

\section{Folded honeycomb lattices}

In this section we describe the general method by which the free-fermion theory can be solved on homogeneous curved lattices. By an homogeneous lattice we mean one in which the coordination number remains constant for all the vertices. These are honeycomb lattices inscribed on the tetrahedron, the octahedron or the icosahedron. To illustrate the method we take the particular case of a generic honeycomb lattice inscribed on the tetrahedron. On topological grounds, the tetrahedron is an orbifold, i.e. a manifold with several singular points. There exists a particular set of coordinates which maps this orbifold into a bounded region of the two-dimensional plane, as shown in fig. 5 [7]. When such a coordinate system is chosen the lattice can be unfolded on the plane, bearing in mind the appropriate identifications of points.

We want to solve the hamiltonian of coupled fermion oscillators (16),

$$
H=\gamma \sum_{\langle i, j\rangle} a_{i}^{+} a_{j},
$$

where the sum is now over pairs of nearest neighbors $i, j$ on the folded honey- 
comb lattice. Suppose that we introduce a would-be eigenvector

$$
\Psi=\sum_{i} f_{i} a_{i}^{+}|O\rangle
$$

then, under the action of the hamiltonian we have

$$
H \Psi=\gamma \sum_{i} \sum_{\langle i, j\rangle} f_{j} a_{i}^{+}|O\rangle,
$$

where the sum over $j$ is over nearest neighbors of the $i$ vertex. We have a solution of the eigenvalue problem if and only if the quantity

$$
\frac{1}{f_{i}} \sum_{\langle i, j\rangle} f_{j}=\lambda
$$

is constant over the lattice. In the particular coordinate system which maps the tetrahedron on the region of fig. 5 , it is not difficult to think of functions which satisfy (29), with $\lambda$ being a constant. Actually, the most general solution of this difference equation is given by a combination of exponential functions. We have to require, however, that the given combination be single valued on the tetrahedron. At this point, it proves useful to turn for a moment to the boundary value problem on the continuum.

Suppose that we were looking for single valued and differentiable functions on the region of fig. 5, with all pertinent identifications made. The set of plane waves allows us to build a complete set matching all boundary conditions implied by the identifications. These are, in units in which the side of the triangular faces is $L$,

$$
\begin{aligned}
\Psi(0, y) & =\Psi(0,-y), \\
\boldsymbol{n} \cdot \nabla \Psi(0, y) & =-\boldsymbol{n} \cdot \nabla \Psi(0,-y), \\
\Psi\left(\frac{1}{2} \sqrt{3} L, \frac{1}{2} L+y\right) & =\Psi\left(\frac{1}{2} \sqrt{3} L, \frac{1}{2} L-y\right), \\
\boldsymbol{n} \cdot \nabla \Psi\left(\frac{1}{2} \sqrt{3} L, \frac{1}{2} L+y\right) & =-\boldsymbol{n} \cdot \nabla \Psi\left(\frac{1}{2} \sqrt{3} L, \frac{1}{2} L-y\right), \\
\Psi(x, y) & =\Psi(x, y+2 L),
\end{aligned}
$$

where $\boldsymbol{n}$ denotes always the normal unit vector pointing outwards the given boundary. The first two boundary conditions can be satisfied at once by taking

$$
\Psi \sim \cos (\boldsymbol{k} \cdot \boldsymbol{r}) .
$$

The requirement of periodicity (34) implies that

$$
k_{y}=\frac{\pi}{L} n, \quad n \in \mathbb{Z} .
$$

Finally, it is easily seen that (32) and (33) are satisfied provided that

$$
\frac{1}{2} \sqrt{3} k_{x}+\frac{1}{2} k_{y}=\frac{\pi}{L} m, \quad m \in \mathbb{Z},
$$


which gives the constraint

$$
k_{x}=\frac{2}{\sqrt{3}} \frac{\pi}{L}\left(m-\frac{1}{2} n\right) .
$$

We have found, therefore, a complete set $\left\{\Psi_{(m, n)}\right\}$, with

$$
\begin{gathered}
\Psi_{(m, n)}=\cos \left(\boldsymbol{k}^{(m, n)} \cdot \boldsymbol{r}\right), \\
\boldsymbol{k}^{(m, n)}=\frac{2 \pi}{\sqrt{3} L}\left(m-\frac{1}{2} n\right) \boldsymbol{e}_{x}+\frac{\pi}{L} n \boldsymbol{e}_{y}, \quad m, n \in \mathbb{Z}^{+},
\end{gathered}
$$

for the differentiable functions on the tetrahedron.

Going back to our original problem, one finds that the functions $\Psi_{(m, n)}$ are not by themselves solutions to eq. (29). However, it is now an easy task to produce solutions for the eigenvalue problem on the lattice. One has first to split artificially the points in fig. 5 in two categories, say black points and blank points, depending on the orientation of the adjacent links as shown in fig. 5 . Then, one may form a function $\Psi^{(1)}$ on the lattice of the type (27) with

$$
\begin{array}{ll}
f_{i}^{(1)}=\exp \left(+i \boldsymbol{r}_{i} \cdot \boldsymbol{k}^{(m, n)}\right) & \text { for black points, } \\
f_{i}^{(1)}=\exp \left(-i \boldsymbol{r}_{i} \cdot \boldsymbol{k}^{(m, n)}\right) & \text { for blank points. }
\end{array}
$$

Alternatively, one may also form a function $\Psi^{(2)}$ with

$$
\begin{array}{ll}
f_{i}^{(2)}=\exp \left(-i \boldsymbol{r}_{i} \cdot \boldsymbol{k}^{(m, n)}\right) & \text { for black points, } \\
f_{i}^{(2)}=\exp \left(+i \boldsymbol{r}_{i} \cdot \boldsymbol{k}^{(m, n)}\right) & \text { for blank points. }
\end{array}
$$

It is obvious that either of these two choices gives rise to a single valued function for the lattice on the tetrahedron. On the other hand, by application of the hamiltonian on $\Psi^{(1)}$ we get

$$
\begin{aligned}
H \Psi^{(1)} & =\gamma \sum_{i \bullet} \sum_{\langle i, j\rangle} \exp \left(-i \boldsymbol{r}_{j} \cdot \boldsymbol{k}^{(m, n)}\right) a_{i}^{+}|O\rangle+\gamma \sum_{i \circ} \sum_{\langle i, j\rangle} \exp \left(i \boldsymbol{r}_{j} \cdot \boldsymbol{k}^{(m, n)}\right) a_{i}^{+}|O\rangle \\
& =\gamma \sum_{i \bullet} \exp \left(-i \boldsymbol{r}_{i} \cdot \boldsymbol{k}^{(m, n)}\right) \sum_{j} \exp \left(-i \boldsymbol{u}_{j} \cdot \boldsymbol{k}^{(m, n)}\right) a_{i}^{+}|O\rangle \\
& +\gamma \sum_{i \circ} \exp \left(+i \boldsymbol{r}_{i} \cdot \boldsymbol{k}^{(m, n)}\right) \sum_{j} \exp \left(+i \boldsymbol{v}_{j} \cdot \boldsymbol{k}^{(m, n)}\right) a_{i}^{+}|O\rangle
\end{aligned}
$$

where $\left\{\boldsymbol{u}_{j}\right\}$ and $\left\{\boldsymbol{v}_{j}\right\}$ are the two triads mentioned in the previous section. The quantity

$$
\sum_{j} \exp \left(-i \boldsymbol{u}_{j} \cdot \boldsymbol{k}^{(m, n)}\right)=\sum_{j} \exp \left(i \boldsymbol{v}_{j} \cdot \boldsymbol{k}^{(m, n)}\right)=\lambda_{(m, n)}
$$

is constant over the lattice, so that $\Psi^{(1)}$ is mapped into $\Psi^{(2)}$ by the action of the hamiltonian, and vice versa. In this way we have built, for each pair $(m, n)$, a two-dimensional space which is held invariant under the action of $H$. The 
eigenvalues of this operator can be expressed, therefore, in the form

$E_{(m, n)}= \pm \gamma\left|\lambda_{(m, n)}\right|= \pm \gamma \sqrt{1+4 \cos ^{2} \frac{1}{2} \sqrt{3} k_{x}^{(m, n)}+4 \cos \frac{1}{2} \sqrt{3} k_{x}^{(m, n)} \cos \frac{3}{2} k_{y}^{(m, n)}}$.

At this point, we may ask whether all the momenta which satisfy the boundary conditions in the continuum are compatible with the quantization conditions imposed by the lattice. These arise from the fact that, under certain translations, the lattice maps into itself. An independent set of them is given by the transformations $2 P\left(T_{1}+T_{2}\right)$ and $2 P\left(-T_{1}+2 T_{2}\right)$, where $P$ is a positive integer equal to $\frac{2}{3}$ times the number of hexagons along the $x$-direction. The projections of the allowed momenta onto these two vectors are quantized in the form

$$
\begin{gathered}
2 P \boldsymbol{k} \cdot\left(\boldsymbol{T}_{1}+\boldsymbol{T}_{2}\right)=2 \pi p, \quad p \in \mathbb{Z}, \\
2 P \boldsymbol{k} \cdot\left(-\boldsymbol{T}_{1}+2 \boldsymbol{T}_{2}\right)=2 \pi q, \quad q \in \mathbb{Z} .
\end{gathered}
$$

The momenta (40) found in the continuum satisfy automatically these conditions. This is easily seen after adjusting properly the length $L$ of the sides of the triangular faces to its lattice dimension, $3 P$. We end up with the outcome that the allowed momenta on the continuum fill up the first Brillouin zone of the lattice. In general, every two opposite momenta give rise to two solutions with opposite energy on the lattice. There are only a few exceptions to this rule, corresponding to those momenta sitting on the boundary of the first Brillouin zone, in which the lattice actually identifies modes corresponding to different momenta. Only in such cases there is one mode for each pair of opposite points in the reciprocal lattice. This explains why in all the honeycomb lattices inscribed in the tetrahedron the number of zero modes is two, one for each of the two independent Fermi points. We give in table 1 the spectrum of the independent modes contained in the first Brillouin zone, in the particular case $P=2$ which corresponds to the lattice of fig. 5 (we set $\gamma=-1$ ). The energy eigenvalues coincide precisely with those obtained by numerical diagonalization of the lattice hamiltonian, represented in the top diagram of fig. 7 .

The method we have just illustrated in the case of the tetrahedron can be applied to solve the spectrum of honeycomb lattices inscribed in any of the remaining orbifolds, namely the octahedron and the icosahedron. The lesson that we learn by following this approach is that the eigenfunctions of the curved lattice are given by momenta still lying in the first Brillouin zone, in which some points have been identified according to the symmetries of the lattice unfolded on the plane. In the case of the icosahedron, however, one may devise more economic techniques in order to predict the relevant properties for solid-state applications. It is possible, as we will see in what follows, to extract the essentials of the method described above, in order to model the properties of the modes near the Fermi points to a high degree of approximation. 
TABLE 1

Spectrum of the hopping hamiltonian for the lattice in fig. 5

\begin{tabular}{|c|c|c|}
\hline$k_{x}$ & $k_{y}$ & $E$ \\
\hline 0 & 0 & -3 \\
\hline $\begin{array}{c}\pi / 3 \sqrt{3} \\
\pi / 6 \sqrt{3} \\
-\pi / 6 \sqrt{3}\end{array}$ & $\begin{array}{l}\pi / 6 \\
\pi / 6\end{array}$ & $\pm(1+\sqrt{3})$ \\
\hline$\pi / 2 \sqrt{3}$ & $\begin{array}{l}\pi / 6 \\
\pi / 3\end{array}$ & $\pm \sqrt{5}$ \\
\hline$-\pi / 2 \sqrt{3}$ & $\pi / 6$ & \\
\hline $\begin{array}{c}2 \pi / 3 \sqrt{3} \\
\pi / 3 \sqrt{3} \\
-\pi / 3 \sqrt{3}\end{array}$ & $\begin{array}{l}\pi / 3 \\
\pi / 3\end{array}$ & \pm 2 \\
\hline $\begin{array}{c}5 \pi / 6 \sqrt{3} \\
2 \pi / 3 \sqrt{3} \\
\pi / 6 \sqrt{3} \\
-5 \pi / 6 \sqrt{3} \\
-2 \pi / 3 \sqrt{3} \\
-\pi / 6 \sqrt{3}\end{array}$ & $\begin{array}{l}\pi / 6 \\
\pi / 3 \\
\pi / 2 \\
\pi / 6 \\
\pi / 3 \\
\pi / 2\end{array}$ & $\pm \sqrt{2}$ \\
\hline $\begin{array}{c}\pi / \sqrt{3} \\
\pi / 2 \sqrt{3} \\
-\pi / 2 \sqrt{3}\end{array}$ & $\begin{array}{l}\pi / 2 \\
\pi / 2\end{array}$ & \pm 1 \\
\hline $\begin{array}{c}\pi / \sqrt{3} \\
-\pi / \sqrt{3}\end{array}$ & $\begin{array}{c}\pi / 3 \\
2 \pi / 3 \\
\pi / 3\end{array}$ & 1 \\
\hline $\begin{array}{c}7 \pi / 6 \sqrt{3} \\
\pi / 3 \sqrt{3} \\
-7 \pi / 6 \sqrt{3}\end{array}$ & $\begin{array}{c}\pi / 6 \\
2 \pi / 3 \\
\pi / 6\end{array}$ & $\pm(\sqrt{3}-1)$ \\
\hline $\begin{array}{l}4 \pi / 3 \sqrt{3} \\
2 \pi / 3 \sqrt{3}\end{array}$ & $2 \pi / 3$ & 0 \\
\hline
\end{tabular}

\section{Effective field theories in the continuum limit}

In this section, rather than pursuing the exact diagonalization of the hamiltonian (26) we undertake the formulation of effective field theories describing the low-energy excitations of the folded honeycomb lattices at half-filling. The analysis of the previous section indicates the general way to proceed, in a manner which makes possible the treatment of the more complicated lattice on the icosahedron. The resolution of the honeycomb lattice on the tetrahedron shows that the formal expression of the dispersion relation remains untouched in the curved lattice, and susceptible of being considered in the continuum limit to 
produce a simple field theory. Taking the continuum limit is a way of amplifying the structure of the levels infinitely close to each of the Fermi points. For this reason, the states of the effective field theory are attached to any of the two independent Fermi points, giving rise in the case of the planar lattice to the spectrum of two uncoupled Dirac spinors. Regarding folded lattices in which the two sublattices of black and blank points are exchanged by going around a conical singularity, the admissible wavefunctions are made of pairs of plane waves with opposite momenta. We have already applied this construction for the lattice on the tetrahedron, and it turns out to be also pertinent for the lattice on the icosahedron. In momentum space the inversion with respect to the origin exchanges the two independent classes of Fermi points. It becomes clear that, for the mentioned honeycomb lattices, the states of the theory have to accommodate into the spectrum of two coupled Dirac spinors.

To understand the nature of the interaction between the two spinors we may have a deeper look at the process of diagonalization of the lattice hamiltonian. The boundary conditions imposed in (30)-(33) are a resort to obviate the fact that, in principle, two charts are needed to cover each of the conical singularities. Focusing on the singularity at the origin, for instance, two appropriate local coordinate systems are depicted in fig. 6 . In each separate coordinate patch the two wavefunctions

$$
\begin{aligned}
& \Psi_{\bullet}=\sum_{i \bullet} \exp \left(i \boldsymbol{k} \cdot \boldsymbol{r}_{i}\right) a_{i}^{+}|O\rangle, \\
& \Psi_{\circ}=\sum_{i \circ} \exp \left(i \boldsymbol{k} \cdot \boldsymbol{r}_{i}\right) a_{i}^{+}|O\rangle
\end{aligned}
$$

span a two-dimensional invariant subspace in which the effective hamiltonian is the same as in (19),

$$
\mathcal{H}_{+}=\left(\begin{array}{cc}
0 & \gamma \sum_{j} \exp \left(i \boldsymbol{k} \cdot \boldsymbol{u}_{j}\right) \\
\gamma \sum_{j} \exp \left(i \boldsymbol{k} \cdot \boldsymbol{v}_{j}\right) & 0
\end{array}\right) .
$$

A similar pair of wavefunctions with the opposite momentum gives rise to a second effective hamiltonian

$$
\mathcal{H}_{-}=\left(\begin{array}{cc}
0 & \gamma \sum_{j} \exp \left(-i \boldsymbol{k} \cdot \boldsymbol{u}_{j}\right) \\
\gamma \sum_{j} \exp \left(-i \boldsymbol{k} \cdot \boldsymbol{v}_{j}\right) & 0
\end{array}\right) .
$$

The respective regions to the left of the two coordinate patches are in correspondence by an appropriate map, and the important point is that under this mapping every vector in the tangent space suffers a rotation of $\pi$. This applies 


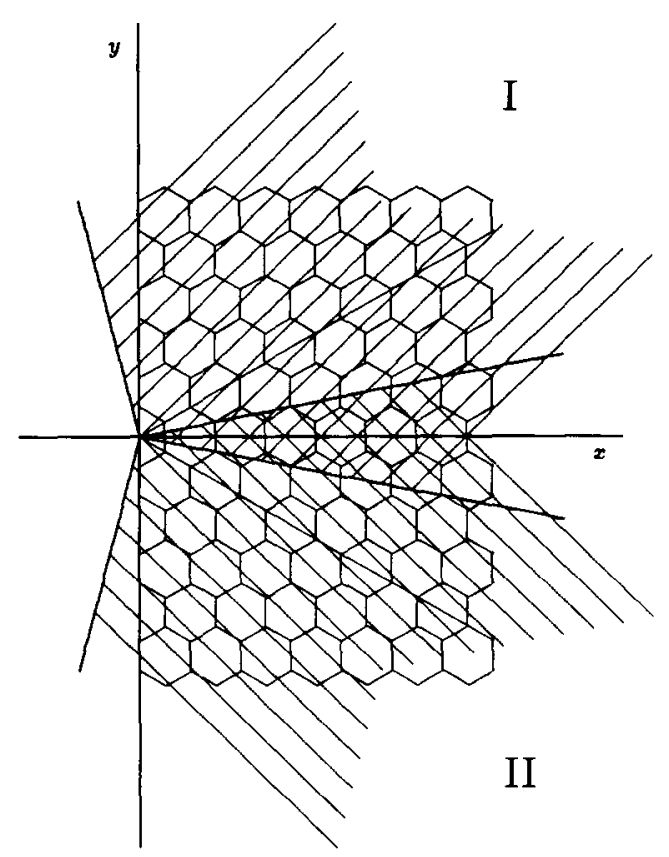

Fig. 6. The conical singularity at the origin. The two different shaded regions represent two local coordinate systems needed to cover the tetrahedron vertex.

in particular to the momenta, so that when going from the left of region I to the left of region II in fig. 6 the two effective hamiltonians (50) and (51) are exchanged.

In the continuum limit, the rotation of the momenta has the following consequences. A momentum $\boldsymbol{k}$ about the Fermi level like (23) is mapped into

$$
-\boldsymbol{k}=-\frac{4 \pi}{3 \sqrt{3}} \boldsymbol{e}_{x}-\boldsymbol{\delta} \boldsymbol{k}
$$

This implies that an operator like $\mathcal{H}_{+}$in the continuum limit

$$
\lim _{a \rightarrow 0} \mathcal{H}_{+} / a=-\left.\frac{3}{2} \gamma \boldsymbol{\sigma}^{\mathrm{T}} \cdot \boldsymbol{\delta} \boldsymbol{k}\right|_{\boldsymbol{k}=(4 \pi / 3 \sqrt{3}) \boldsymbol{e}_{x}}
$$

goes by the mentioned mapping into the operator

$$
\lim _{a \rightarrow 0} \mathcal{H}_{-} / a=\left.\frac{3}{2} \gamma \boldsymbol{\sigma}^{\mathrm{T}} \cdot \boldsymbol{\delta} \boldsymbol{k}\right|_{\boldsymbol{k}=-(4 \pi / 3 \sqrt{3}) \boldsymbol{e}_{x}} .
$$

The rotation suffered by $\boldsymbol{\delta} \boldsymbol{k}$ is of the kind produced by the spin connection for the curvature accumulated at the conical singularity. However, the change of Fermi point when going from (53) to (54) has a different character, and has to be dealt with separately by means of a different connection. 
The mapping between the two local coordinate systems I and II exchanges excitations which are bounded respectively to two independent Fermi points. In the continuum limit this means the exchange of the two Dirac spinors, and this kind of twist can be realized by a proper connection in the internal space of these two fields. In general, the connection can be given support on a set of disjoint segments linking pairs of neighboring singularities on the two-dimensional surface. On the tetrahedron and the icosahedron we may form, respectively, two and six of such cuts, across of which the rotation in the internal space of the two spinors takes place. More physically, the rotation can be implemented by inserting a line of magnetic flux at each of the conical singularities. The flux has to be nonabelian and properly adjusted to produce the twist by going around the puncture. A correct parametrization is achieved by introducing the SU(2)valued connection

$$
\boldsymbol{A} \equiv \boldsymbol{A}^{(a)} \tau^{(a)}, \quad a=1,2,3,
$$

with $\left\{\tau^{(a)}\right\}$ being the three Pauli matrices. The only nonvanishing component may be taken to be, in local polar coordinates around each puncture,

$$
A_{\phi}=\frac{\Phi}{2 \pi} \tau^{(2)} \text {. }
$$

By setting the magnetic flux to $\Phi=\frac{1}{2} \pi$, the nonabelian phase acquired by the doublet of spinors after going around each conical singularity is, as required,

$$
\exp (i \oint \boldsymbol{A})=\left(\begin{array}{cc}
0 & 1 \\
-1 & 0
\end{array}\right) \text {. }
$$

This picture implies the existence of a fictitious magnetic monopole inside the surface. Its charge $g$ can be computed by adding up the individual fluxes of all the lines

$$
g=\frac{1}{4 \pi} \sum_{i=1}^{N} \frac{1}{2} \pi=\frac{1}{8} N
$$

$N$ being the number of conical singularities on the surface. It is worth mentioning that the values of $g$ required for the tetrahedron and the icosahedron are $\frac{1}{2}$ and $\frac{3}{2}$, respectively, and therefore compatible with the standard quantization condition of the monopole charge [8].

To summarize, we have developed a picture in which the continuum limit for honeycomb lattices on the tetrahedron and the icosahedron at half-filling is given by the effective field theory of a doublet of spinors interacting with the curvature and color magnetic fields accumulated at a certain number of conical singularities. Although the exact resolution of this model is beyond the scope of the present paper, it is possible to show with much less effort that it reproduces the low-energy spectra and correct numbers of zero modes for the mentioned lattices in the limit of large number of points. As a first approximation, one 
may consider the model in which both curvature and magnetic field are made uniform over the two-dimensional surface. Then the system becomes that of a couple of spinors on the sphere with magnetic monopole field. The spectrum is obtained by solving the eigenvalue problem for the covariant Dirac operator [9],

$$
i \sigma^{a} e_{a}^{\mu}\left(\nabla_{\mu}-i A_{\mu}\right) \Psi_{n}=\varepsilon_{n} \Psi_{n}, \quad a, \mu=1,2,
$$

where $e_{a}^{\mu}$ is the zweibein for the sphere and, in spherical coordinates,

$$
\begin{aligned}
& \nabla_{\theta}=\partial_{\theta}, \\
& \nabla_{\phi}=\partial_{\phi}-\frac{1}{4}\left[\sigma^{1}, \sigma^{2}\right] \cos \theta, \\
& A_{\theta}=0, \\
& A_{\phi}=g \cos \theta \tau^{(2)} .
\end{aligned}
$$

The Dirac operator can be diagonalized by introducing the angular momentum of the whole system made of spinor fields, magnetic field and curvature. The total angular-momentum operators turn out to be, for the lower spinor component $\Psi_{\downarrow}$,

$$
\begin{aligned}
J_{ \pm}= & \pm \exp ( \pm i \phi) \nabla_{\theta}+i \exp ( \pm i \phi) \frac{\cos \theta}{\sin \theta}\left(\nabla_{\phi}-i g \cos \theta \tau^{(2)}\right) \\
& -\exp ( \pm i \phi) \sin \theta\left(\frac{1}{2}-g \tau^{(2)}\right), \\
J_{z}= & -i\left(\nabla_{\phi}-i g \cos \theta \tau^{(2)}\right)-\cos \theta\left(\frac{1}{2}-g \tau^{(2)}\right) .
\end{aligned}
$$

For the upper component $\Psi_{\uparrow}$, the corresponding operators are similar except for a change of sign in front of the $\frac{1}{2}$ fractions. By squaring the Dirac operator, each of the spinor components comes to obey the equation, with respective angularmomentum operators,

$$
\left(J^{2}+\frac{1}{4}-g^{2}\right) \Psi_{n}=\varepsilon_{n}^{2} r^{2} \Psi_{n},
$$

where $r$ parametrizes the radius of the sphere. The spectrum is given in terms of the angular-momentum quantum number $j$

$$
\varepsilon_{j}^{2} r^{2}=j(j+1)+\frac{1}{4}-g^{2}=\left(j+\frac{1}{2}\right)^{2}-g^{2} .
$$

As is well known, there is a minimum value of the angular momentum $j$ dictated by $g$, so that the number of zero modes in the spectrum depends exclusively on the value of the monopole charge.

Let us specialize now to the models corresponding, respectively, to the tetrahedron and the icosahedron. In the first case the monopole charge required is $g=\frac{1}{2}$, and the model predicts the existence of two zero modes, one for each spinor component, in the continuum limit of the honeycomb lattice at halffilling. This result can be tested with the spectra obtained by numerical diagonalization of the hamiltonian (26). Let us call generically a honeycomb lattice 

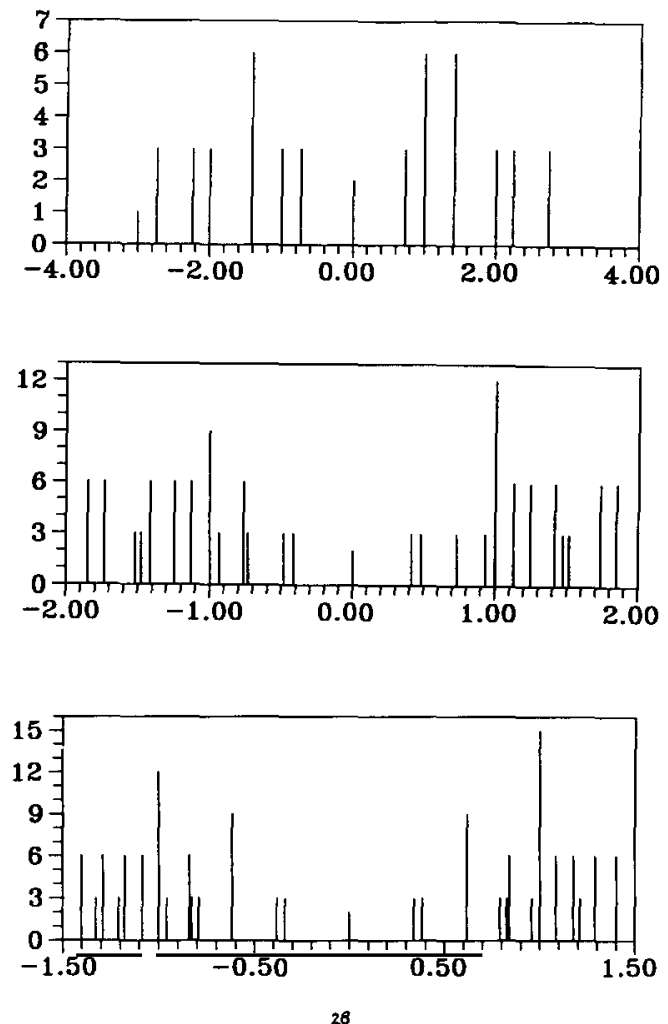

Fig. 7. Spectra of different honeycomb lattices on the tetrahedron. The horizontal axis stands for energy values and the height of the segment gives in each case the degeneracy of the multiplet. The three diagrams correspond, respectively, to the lattices $\Theta_{48}, \Theta_{192}$ and $\Theta_{300}$.

inscribed on the tetrahedron by $\Theta_{m}, m$ being the total number of points in the lattice. These lattices can be ordered along a sequence of increasing $m$, whose general term is of the form $\Theta_{12(n+1)^{2}}, n \in \mathbb{Z}^{+}$. We have depicted the result of the numerical diagonalization of three of these lattices in fig. 7 (we take units in which $\gamma=-1$ ). In all cases, two states appear with an energy compatible with zero within the computer precision, showing that our mean-field approximation already produces the correct number of zero modes. Furthermore, from $\Theta_{192}$ on the next low-energy states are given by two close triplets above the Fermi level and two other with the opposite energy. This bears a reasonable agreement with what is expected from the formula (67). The highest dimension of an irreducible representation for the tetrahedron symmetry group is three and, consequently, a significant departure from spherical symmetry develops above $j=1$ in the spectrum.

We deal similarly with the honeycomb lattices folded on the icosahedron. The effective field theory demands now a monopole charge $g=\frac{3}{2}$. Relying 
again on the spherical approximation to compute the number of zero modes, we come out with the theoretical prediction that there should be a couple of triplets lying at zero energy, in the continuum limit. We denote the fullerene lattices by $\mathrm{C}_{m}$ according to the total number of lattice points $m$. The sequence with increasing number of points is given by the general term $\mathrm{C}_{60(n+1)^{2}}, n \in \mathbb{Z}^{+}$. We have represented in fig. 8 the spectra obtained by numerical diagonalization of three such lattices (we have set again $\gamma=-1$ ). Although we do not find as before any zero modes from the start, there is clear evidence that the couple of triplets close to zero energy approach asymptotically the origin of the energy scale in the limit of large lattices. The levels given by the formula (67) are in good agreement with those in the numerical spectra up to the point in which the highest dimension of an irreducible representation of the icosahedron symmetry group is reached. This happens for $j=2$. The levels next to the couple of quintuplets at each side of the spectrum are a couple of quadruplets and other of triplets, which may be thought as arising from the split of two $j=3$ multiplets by breaking down to the icosahedron symmetry group.

The results of the numerical diagonalization of the lattice hamiltonian (26) support the correctness of the effective field theory developed to account for the continuum limit of the curved lattices. Let us mention, finally, that there is a similar sequence of honeycomb lattices folded on the octahedron. In all of them one may define consistently two sublattices of black and blank points, respectively, over the whole surface. According to our picture, the continuum limit for these lattices at half-filling should be given by a field theory on the octahedron in the absence of magnetic field. The inspection of the numerical spectra for these lattices shows that none of them has any mode sitting at zero energy, nor this value is approached asymptotically in the continuum limit. It is reassuring to find that this is, in fact, the prediction obtained in the framework of the theoretical model after switching off the magnetic field.

\section{Summary and prospects}

The leitmotif of this paper was to explain the details of how a continuum model can be used to study the electronic and elastic properties of the fullerene molecules [5]. Along the way we have found some interesting points that we will highlight and comment here.

This work is based on two fundamental points. The first one is the existence of isolated Fermi points instead of lines in the graphite molecule at half-filling. This leads to the formulation of a continuum limit for describing the spectrum of electronic excitations around any of these points. We have seen that the particular geometry of the lattice determines the continuum model to be that of two massless, independent, free, Dirac spinors. 

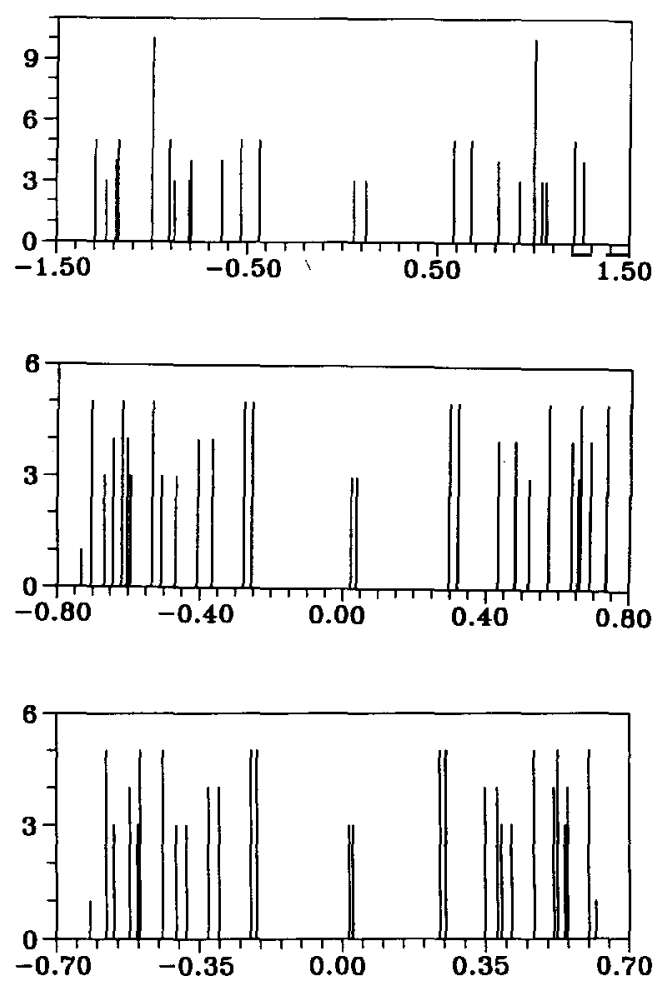

29

Fig. 8. Spectra of honeycomb lattices on the icosahedron. Energy eigenvalues are plotted in the horizontal axis and the multiplet degeneracy is given along the vertical direction as in fig. 7 . The diagrams correspond, respectively, to the lattices $C_{240}, C_{960}$ and $C_{1500}$.

The second basic fact in this paper is the existence of bigger fullerene molecules derived from a given geometry and the regularity found in their spectrum of excitations around the Fermi level. That is what allows us to propose the validity for them of the continuum limit found in the - infinite - graphite lattice.

Once we know the properties of the flat graphite lattice, the next interesting point refers to the boundary conditions that can be imposed on the lattice without destroying its main features. At this respect it is worth noticing that the standard procedure to study the properties of Bravais lattices assumes most of the times the choice of Von Karman, i.e. periodic, boundary conditions. While it is obvious that any choice of flat boundary conditions should not alter the main properties of the bulk lattice, we must be careful when considering boundary conditions of the type described in sect. 3. Despite the innocent presentation of fig. 5 , the identifications done to form the polyhedra are curving the lattice - as is clear from fig. 6 - and this is a nontrivial operation. The simplest way to look at it is to think on the way that curvature is induced on the hexagonal tes- 
sellation. This is done by substituting some hexagons by $n$-gons with $n$ less than six. Such substitution will, in general, induce frustration on the Bravais lattice that underlies the hexagonal lattice. In particular, for odd $n$, the two triangular sublattices are interchanged. It is then not trivial that we could successfully complete the study of the tetrahedron.

Before leaving the subject of the boundary conditions, let us mention some results concerning the simplest situation, namely, the case of a graphite sheet with periodic boundary conditions. When the lattice is folded so as to form a torus, i.e., a compact surface with no intrinsic curvature, one finds that the electronic spectrum is precisely the one predicted by the continuum model: the doubled spectrum of a Dirac spinor in a plane with doubly periodical boundary conditions. What we observe numerically is a structure of quadruplets (one doublet for each Dirac spinor) equally spaced in energy. We end this comment by mentioning that cylindrical shapes and tubular structures closed at the extremes with conical singularities (capsules) have been described in ref. [12].

The phenomenological model described in sect. 4 summarizes all the features discussed so far. We are led to study two Dirac spinors on compact surfaces with curvature singularities and edges of frustration. The sphere takes into account the compactness and curvature of the lattice, and the monopole mimics the frustration.

We envisage various directions in which this work can be continued. The first concerns the statistical mechanics applications. We have seen how, starting from the fullerene molecules, we came to the study of an entirely new family of two-dimensional lattices where one can solve the spectrum of the hopping hamiltonian: honeycomb lattices folded and wrapped around truncated regular polyhedra. Any exactly solvable model in statistical mechanics has some interest on its own, regardless of its immediate applicability to physical problems. In our case, we came the other way around as we took our models directly from existing physical examples. Let us notice that although some of this lattices have already appeared in the literature $[10,11]$, they were formed by a fixed number of points. The main novelty here lies in the fact that our lattices can grow while preserving the coordination and the global symmetry group so that it makes sense to study the continuum and thermodynamical limits. We then have twodimensional statistical models defined on curved, compact surfaces. Work on the study of the thermodynamic limit of two-dimensional models such as the Ising or Hubbard models defined in the new lattices is currently in progress.

As for the solid-state implications, the universal character of the method proposed allows its immediate applicability to the study of the electronic spectrum of all kind of fullerenes existing (as the family of the elliptical $\mathrm{C}_{70}$ ) or proposed. Among those, the geometries with negative curvature [13] may present new challenges as they live in curved but noncompact surfaces. The geometries proposed so far, however, have the advantage of building a three-dimensional 
lattice, so that the problem needs only to be solved within one unit cell. The existence of odd-numbered rings, which now are heptagons, exchanges the two sublattices of the graphite structure. Thus, following the analysis of this paper, a fictitious gauge field needs to be introduced. The detailed nature of this field is postponed to a future publication. On the other hand, the fact that the square of the Dirac equation is the Laplace equation, and the use of periodic boundary conditions, allows us to make some general remarks about the spectrum of these systems [5].

Finally, we leave out of this paper a discussion of the elastic modes of these molecules. For simple central force models, the projection of the structure on a plane, and the imposition of nontrivial boundary conditions can be generalized in a straightforward way. The main difference is that the long-wavelength acoustical modes are related to the center of the Brillouin zone of the flat graphite sheets. For these excitations, frustration does not play the same striking role as at the corners of the Brillouin zone. Thus, we expect that these modes will be well described by the standard theory of elasticity of curved shells.

It is a pleasure to thank G. Sierra for interesting discussions on the subject of the hopping hamiltonian. M.A.H.V. thanks the department of Física Teórica of the Universidad Autónoma of Madrid for financial support during the course of this work. This work has been partially supported by the CICyT (Spain).

\section{References}

[1] H.W. Kroto, J.R. Heath, S.C. O'Brien, R.F. Curl and R.E. Smalley, Nature 318 (1985) 162

[2] R.F. Curl and R.E. Smalley, Sci. Am. 265 (1991) 54; D.R. Huffman, Phys. Today, November (1991) 22; K. Prassides and H. Kroto, Phys. World, April (1992) 44

[3] R.E. Stanton and M.D. Newton, J. Phys. Chem. 92 (1988) 2141

[4] S. Samuel, On the electronic spectrum of $\mathrm{C}_{60}$, CCNY-HEP-92/5, May 1992

[5] J. González, F. Guinea and M.A.H. Vozmediano, Phys. Rev. Lett. 69 (1992) 172

[6] N.W. Ashcroft and N.D. Mermin, Solid state physics (Holt, Rhinehart and Winston, New York, 1976)

[7] S. Deser, R. Jackiw and G. 't Hooft, Ann. Phys. (NY) 152 (1984) 220

[8] S. Coleman, The magnetic monopole fifty years later, in The unity of the fundamental interactions (Plenum, New York, 1983)

[9] N.D. Birrell and P.C.W. Davies, Quantum fields in curved space (Cambridge Univ. Press, Cambridge, 1982)

[10] S. Samuel, The solution of the Ising model in the truncated icosahedron lattice of $\mathrm{C}_{60}$, CCNY-HEP-92/3

[11] S.R. White, Phys. Rev. B45 (1992) 5062

[12] J.W. Mintmire, B.I. Dunlap and C.T. White, Phys. Rev. Lett. 68 (1992) 631

[13] A.L. Mackay and H. Terrones, Nature (London) 352 (1991) 762;

D. Vanderbilt and J. Tersoff, Phys. Rev. Lett. 68 (1992) 511

[14] M.V. Berry, Proc. R. Soc. London A392 (1984) 45 